\title{
Design and Simulation of a Multi-Function MEMS Sensor for Health and Usage Monitoring
}

\author{
Z. Xu ${ }^{1}$, D. Koltsov ${ }^{3}$, A. Richardson ${ }^{1}$, L. $\mathrm{Li}^{2}, \mathrm{M}$. Begbie $^{2}$ \\ ${ }^{1}$ Centre for Microsystems Engineering, Department of Engineering, Lancaster University, Lancaster, LA1 4YR, UK \\ ${ }^{2}$ Institute for System Level Integration, Livingston, EH54 7EG, UK \\ ${ }^{3}$ BREC Solutions, Lancaster, LA1 2DG, UK
}

\begin{abstract}
Health and usage monitoring as a technique for online test, diagnosis or prognosis of structures and systems has evolved as a key technology for future critical systems. The technology, often refereed to as HUMS is usually based around sensors that must be more reliable than the system or structure they are monitoring. This paper proposes a fault tolerant sensor architecture and demonstrates the feasibility of realising this architecture through the design of a dual mode humidity/pressure MEMS sensor with an integrated temperature function. The sensor has a simple structure, good linearity and sensitivity, and the potential for implementation of built-inself-test features. We also propose a re-configurable sensor network based on the multi-functional sensor concept that supports both Normal Operational and Fail Safe Modes. The architecture has the potential to significantly increase system reliability and supports a reduction in the number of sensors required in future HUMS devices. The technique has potential in a wide range of applications, especially within wireless sensor networks.
\end{abstract}

Keyword: HUMS, multi-mode sensing, humidity sensor, pressure sensor, sensor network, sensor reliability, built-inself-test, online test, self-repair, bias superposition, wireless sensor network.

\section{INTRODUCTION}

Health and Usage Monitoring Systems, (HUMS) range from single sensors that monitor use and condition of a utility to sub-systems that contain sensors, processors and algorithms to monitor system or structure integrity, reliability or condition. The traditional applications of HUMS are on aircraft, especially rotorcraft vehicles to improve safety and reduce maintenance cost. One of the early studies of HUMS installed on helicopters is associated with the UK North Sea helicopter operation [1]. In this study, 63 airworthiness related events which led to significant maintenance work were observed. Onboard HUMS successfully detected $69 \%$ of them.

HUMS used in helicopters are reviewed in [2]. These systems typically monitor vibration and measure the impact on rotor components. According to this survey, HUMS is already in wide use. Examples given include the Sikorsky SH-60B Black Hawk[3], S-61 and S-76[4], the EH-101[5], the Boeing CH-47D Chinook[6], MH-47E[7], WAH-64 Apache[8], UH60A Black Hawk[9], and AH-64D Apache[10].

Whilst the efficacy of HUMS on life critical systems has been proven [11, 12], the application of the concept within systems that require lower cost are less well developed. Recently R. Heine and D. Barker developed a HUMS solution within an army eight wheeled vehicle [13]. In this work, an array of sensors and simplified algorithm are used to identify the terrain and its effect on mechanical components on the vehicle. examples of work on HUMS outside of the military and aerospace sectors can be found in [14-18].

Regardless of their application or complexity, most HUMS devices that have been realised to date require in-situ sensors to measure environmental parameters in real time. These sensors normally need to be small enough to be integrated into a HUMS systems through ideally connectorless technologies and support low weight overhead and application within small, difficult to access locations [19]. In applications such as consumer electronics, the price of these sensors is a further critical issue regarding uptake. Micro-Electro-MechanicalSensors (MEMS) are emerging as a natural choice here due to their size, compatibility with monolithic and hybrid integration technologies and inherent robustness.

Even with MEMS technology, guaranteeing the reliability of microsensors for HUMS applications is a challenging task, especially when considering the operational environments. For example, in military ground vehicle systems sensors frequently experience dramatic temperature changes, moisture, dust, shock, etc. An example of the importance of the HUMS device reliability exceeding that of the systems is given in [1] where system failure was not detected due to the failure of a sensor within the HUMS architecture. To ensure the robustness of the sensors used in HUMS, they should have the following features:

- Online self-testable: validation of the raw measurement is essential as the HUMS concept relies on the elimination of false positives and negatives.

- $\quad$ Self-repairing. Most HUMS devices are designed to be permanently assembled within the systems being monitored which is often difficult to access. Failure can invalidate insurance, compromise safety or necessitate immediate and normally extensive repair work. Some form of self-repair or at the minimum a backup mode is needed for most critical applications.

The traditional solution to meet the above requirement is through the use of redundancy. At least three sensors are used to measure one parameter. If the outputs all match one another, the measurement is reliable; if one of them does not match with the others, a sensor failure is logged. However, useable reading could still be provided by the remaining healthy sensors, and maintenance could be suggested to prevent full system failure. The problem of redundancy is it significantly increases the number of sensors to implement the task, which makes it impossible for many applications. For example to equip HUMS on helicopter engine and transmission systems alone needs 3070 different sensors [20]. For wiring systems on aircraft it could easily grow to several hundreds of electrical connectors to several different types of sensors [21]. Supplemental power, communication, processing capabilities requirements and soaring weight, cost and complexity of the system simply overshadow the benefit of installing HUMS.

To improve the reliability of the sensors used in HUMS, we propose a concept of multi-mode sensing combined with a reconfigurable sensor network. In this paper we will demonstrate the design and simulation of a MEMS based Dual-Mode humidity/pressure Sensor (DMS). A temperature measurement can also be integrated. Compared to a traditional redundancy solution the advantages include but not limited to: 
a) a reduction in the number of sensors and system complexity as a DMS can measure several physical parameters concurrently, and

b) a simplification of the system integration architecture, especially self-test as only one test stimuli is required to cover multiple sensing modes.

\section{DESIGN AND MODELLING OF THE DMS}

Relative humidity (RH) and pressure have been selected as the initial physical measurements that would benefit from being integrated due to (a) they are both common and important environmental parameters which often need to be measured together, and (b) both sensors require similar packaging, as the sensing elements have to be exposed to the environment. To integrate these two features we need to exploit structures that have a measureable electrical or physical response to both parameters. In the case of pressure, a common MEMS architecture involves a diaphragm and a sealed cavity. There are two methods to measure the movement/deformation of the diaphragm caused by the pressure difference: capacitive or resistive. The former one uses electrodes deposited on the diaphragm, and the later uses piezoresistive (PZR) integrated into the diaphragm. Again most widely used RH sensors are the capacitive/resistive and PZR devices. The capacitive/resistive type normally contains an active layer as the sensing element. The electrical behaviour of this active layer changes due to the physical/chemical reaction between the layer and water vapour. The change, either in capacitance or resistance, is then measured by embedded electrodes. Typical materials used for the active layer are porous ceramics, polyelectrolytes and metal oxide [22-27]. For the PZR devices, the sensing element is usually a composite structure utilising polymer deposited on silicon (e.g. beam or membrane). When the polymer layer absorbs moisture, it expands and the swelling deforms the composite structure. The deformation is measured by PZR embedded in the silicon layer. Examples of this type of $\mathrm{RH}$ sensor can be found in [28-31]. Commercialized products based on PZR techniques include the HMX2000 series developed by Hygrometrix [32].

For our initial design of a DMS, we used a composite membrane structure with embedded PZR. Compared to the capacitive/resistive type, the electrodes of the PZR are not in direct contact with the active layer, thus increases the stability. The layout of the DMS is given by Fig. 1. In the case of pressure, a square shape membrane is used that generates a reading relative to a sealed cavity. A polymer layer is deposited on the membrane to measure the RH. The DMS has two individual output channels: the $\mathrm{x}$ axis output $V_{x}$ and the $\mathrm{y}$ axis output $V_{y}$. Each channel consists of two piezoresistors placed on the opposite edge of the membrane, parallel and perpendicular to the edge. These two piezoresistors are then connected to a half-Wheatstone bridge (Fig. 2) to give the channel output:

$$
V_{x}=V_{0}\left(\frac{R_{x 1}}{R_{x 1}+R_{x 2}}-\frac{R_{f}}{2 R_{f}}\right)
$$

$$
V_{y}=V_{0}\left(\frac{R_{y 1}}{R_{y 1}+R_{y 2}}-\frac{R_{f}}{2 R_{f}}\right)
$$

Where $V_{0}$ is the supply voltage and $R_{f}$ is the reference resistors.
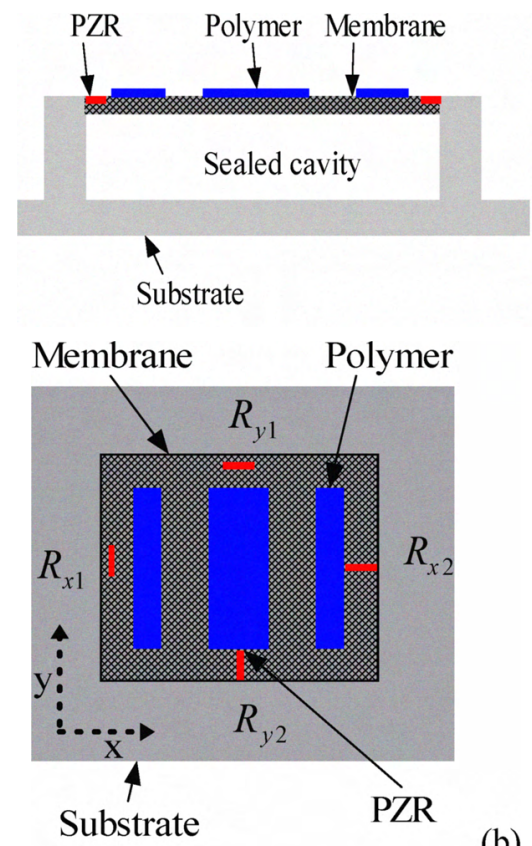

(a)

(b)

Figure 1: (a) Cross view of the DMS. (b) Top view of the DMS. Piezoresistors $R_{\mathrm{x} 1}$ and $\mathbf{R}_{\mathrm{y} 21}$ are parallel to the membrane edges; $R_{x 2}$ and $R_{y 2}$ are perpendicular to the edges.

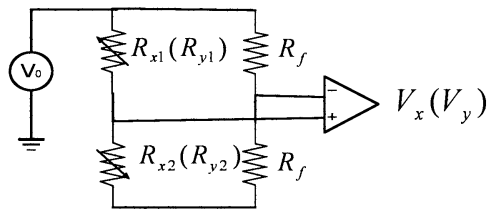

Figure 2: half-Wheatstone bridge used to get individual channel output $V_{x}\left(V_{y}\right)$.

The polymer coating is strip-shaped to give a different coverage ratio on the $\mathrm{x}$ and $\mathrm{y}$ axes. The coverage ratio $(r)$ is defined by $r=w_{p} / w_{m}$, where $w_{p}$ is the width of the polymer and $w_{m}$ is the width of the membrane. Sensitivities of the output to either pressure or RH are related to the coverage ratio. The output voltage can also be described as:

$$
\begin{aligned}
& V_{x}=\alpha_{p x} p+\alpha_{\varphi x} \varphi \\
& V_{y}=\alpha_{p y} p+\alpha_{\varphi y} \varphi
\end{aligned}
$$

Where $p$ is the pressure, $\varphi$ is the RH (\%), $\alpha_{p}$ and $\alpha_{\varphi}$ are the sensitivity to pressure and humidity. As the coverage ratio of the $\mathrm{x}$ and $\mathrm{y}$ axes $\mathrm{r}_{\mathrm{x}}$ and $\mathrm{r}_{\mathrm{y}}$ are not equal, we have $a_{p x} / a_{p y} \neq a_{\varphi x} / a_{\varphi y}$, hence equation (2) is solvable. Which means with two output channels $V_{x}$ and $V_{y}$, we can measure the pressure and the RH concurrently: 


$$
\begin{gathered}
p=\frac{a_{\varphi y} V_{x}-a_{\varphi x} V_{y}}{a_{p x} a_{\varphi y}-a_{p y} a_{\varphi x}} \\
\varphi=\frac{a_{p y} V_{x}-a_{p x} V_{y}}{a_{p x} a_{\varphi y}-a_{p y} a_{\varphi x}}
\end{gathered}
$$

The DMS is modelled and simulated using CoventorWare. The fabrication process of the DMS is summarized as follow:

- 50 um thick p-silicon (100) is prepared as substrate.

- Etch 40 um on the backside of the substrate to create the cavity and the membrane (10 um of thickness).

- Apply ion implementation on the edge of the membrane, topside, to create the PZR.

- Deposit 5 um of polymer on the top of the membrane.

- Etch the polymer as desired sharp.

The material properties and dimensions used to model the DMS are given by Table 1 .

\begin{tabular}{|l|l|}
\hline Density of $\mathrm{Si}(100), \tilde{\mathrm{n}}_{\mathrm{si}}$ & $2.331 \mathrm{e}^{-15} \mathrm{~kg} / \mathrm{um}^{3}$ \\
\hline Young's Modulus of $\mathrm{Si}(100), \mathrm{E}_{\mathrm{si}}$ & $130.18 \mathrm{GPa}$ \\
\hline Possion's ratio of $\mathrm{Si}(100), \tilde{\mathrm{o}}_{\mathrm{si}}$ & 0.278 \\
\hline Density of polymer, $\tilde{\mathrm{n}}_{\mathrm{pol}}$ & $1 \mathrm{e}^{-15} \mathrm{~kg} / \mathrm{um}^{3}$ \\
\hline Young's Modulus of polymer, $\mathrm{E}_{\mathrm{pol}}$ & $7.5 \mathrm{GPa}$ \\
\hline Possion's ratio of polymer, $\tilde{\mathrm{o}}_{\mathrm{pol}}$ & 0.35 \\
\hline $\begin{array}{l}\text { Humidity expansion coefficient of } \\
\text { Polymer, á }\end{array}$ & $1 \mathrm{e}^{-4} / \% \mathrm{RH}$ \\
\hline Residual stress & $0 \mathrm{MPa}$ \\
\hline Width of membrane, $\mathrm{W}_{\mathrm{m}}$ & $500 \mathrm{um}$ \\
\hline Thickness of membrane, $\mathrm{T}_{\mathrm{m}}$ & $10 \mathrm{um}$ \\
\hline Width of side polymer strips & $50 \mathrm{um}$ \\
\hline Width of central polymer strip & $100 \mathrm{um}$ \\
\hline Length of polymer & $400 \mathrm{um}$ \\
\hline Thickness of polymer & $5 \mathrm{um}$ \\
\hline $\begin{array}{l}\text { Offside of the side polymer strips to the } \\
\text { edge of membrane }\end{array}$ & $75 \mathrm{um}$ \\
\hline Coverage ratio of polymer on x axis & 0.4 \\
\hline Coverage ratio of polymer on y axis & 0.8 \\
\hline Width of PZR & $10 \mathrm{um}$ \\
\hline Length of PZR & $50 \mathrm{um}$ \\
\hline Thickness of PZR & $1 \mathrm{um}$ \\
\hline Wheatstone bridge supply voltage $\mathrm{V}_{0}$ & $5 \mathrm{~V}$ \\
\hline Conditioning circuit amplification & 10 \\
\hline
\end{tabular}

Table 1, material properties and parts dimensions

\section{SIMULATION OF THE DMS}

Swelling of the polymer due to absorption of water vapour has a similar effect to thermal expansion. Thus we used the thermal-mechanical solver of CoventorWare to simulate the humidity induced expansion. The residual stress in both the silicon and polymer are dependent on the fabrication process which for this study are set to zero to avoid uncertainty. In production the residual stress due to curing of the polymer layer will lead to an offset in the output voltage. This offset is usually calibrated by experiments and compensated within the DC supply chain.

As expected the pressure and the humidity cause different modes of deformation to the membrane. Fig. 3 shows the differences in the deformed shapes. When only perpendicular pressure is applied, the stress it induces on the $\mathrm{x}$ and $\mathrm{y}$ axes are uniform. However, when humidity is applied, expansion of the polymer mainly induces tensile stress to the membrane, which is related to the polymer coverage ratio. Because of the different coverage ratio on the $\mathrm{x}$ and $\mathrm{y}$ axes $(0.4$ and 0.8$)$, and the 'buffer zone' between the polymer strips, the humidity produces more stress where the $\mathrm{y}$ channel PZR is located than the $x$ channel. Although the sensitivities to pressure of these two channels are also affected by the different coverage ratio, due to the relative small value of Young's Modulus of the polymer, the difference is much smaller. Fig. 4 shows the mises stress distribution on the membrane and the voltage output when (a) $p=0.2 \mathrm{MPa}, \varphi=0 \%$, and (b) $p=0 \mathrm{MPa}, \varphi=100 \%$.

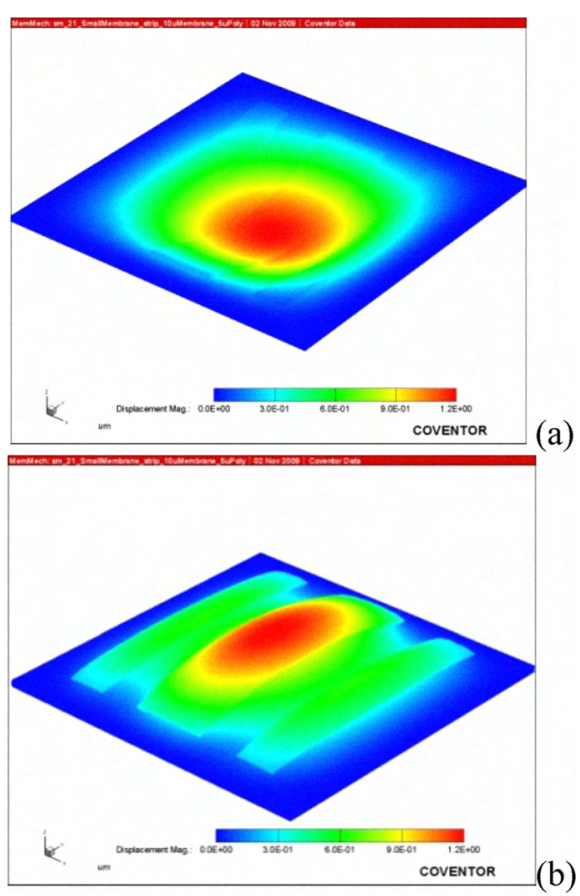

Figure 3, deformation of the membrane when (a) only pressure is applied; and (b) only humidity is applied. Difference comes from the nature of how the stress is changed.

Simulations were carried out with pressure increases from 0 to $0.2 \mathrm{MPa}$ with a step of $0.01 \mathrm{MPa}$, and humidity changes from 0 to $100 \%$, with a step of $5 \%$. The sensitivity map of $\mathrm{x}$ and $\mathrm{y}$ channel outputs are given by Fig. 5. From Fig. 4(b) and Fig. 4(d), by using end-point linear fit, parameters in Equation 2 are found as: $\alpha_{p x}=6.683, \alpha_{\varphi x}=-1.4745, \alpha_{p y}=6.66, \alpha_{\varphi y}=-2.8274$. Using these parameters in equation 3 , the measured pressure $p_{m}$ and RH $\varphi_{m}$ can be obtained. The accuracy of this fitting can be examined by comparing $p_{m}$ and $\varphi_{m}$ with the actual applied value in the simulation $\left(p_{a}\right.$ and $\left.\varphi_{a}\right)$. The fitting error $e$ as a percentage is defined by:

$$
e_{p}=\left|\frac{p_{a}-p_{m}}{p_{a}}\right| \times 100 \quad e_{\varphi}=\left|\frac{\varphi_{a}-\varphi_{m}}{\varphi_{a}}\right| \times 100
$$

The maximum errors found by using the above parameters are $2.8 \%$ in the pressure measurement, when $\mathrm{p}=0.2 \mathrm{MPa}$ and 
$\varphi=100 \%$, and $2.72 \%$ in $\mathrm{RH}$ measurement when $\mathrm{p}=0 \mathrm{MPa}$ and $\varphi=5 \%$. This can be improved by using a more accurate fitting algorithm such as least-square to find the equation parameters.

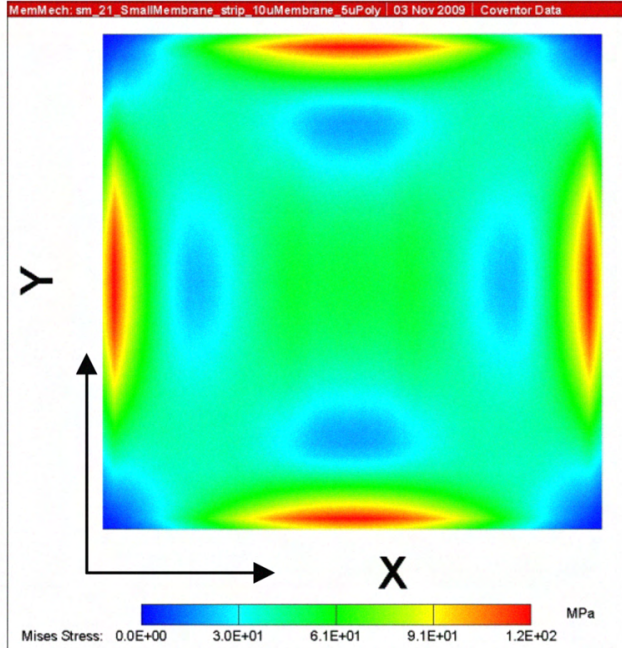

(a)

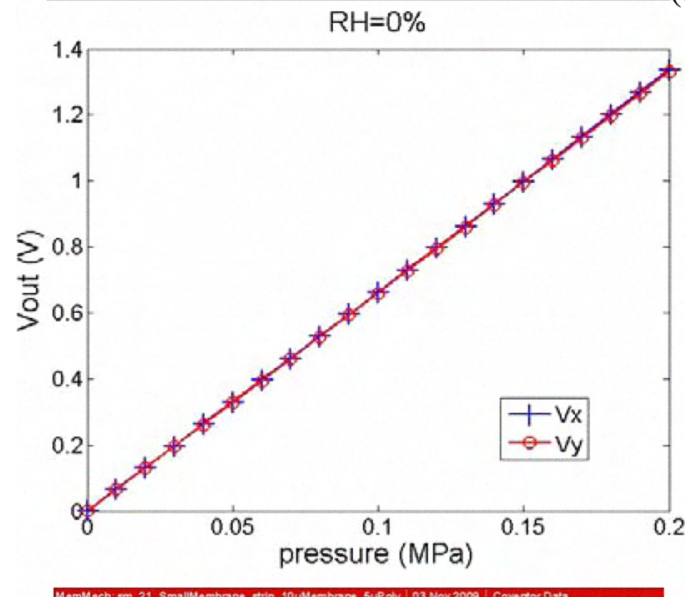

(b)

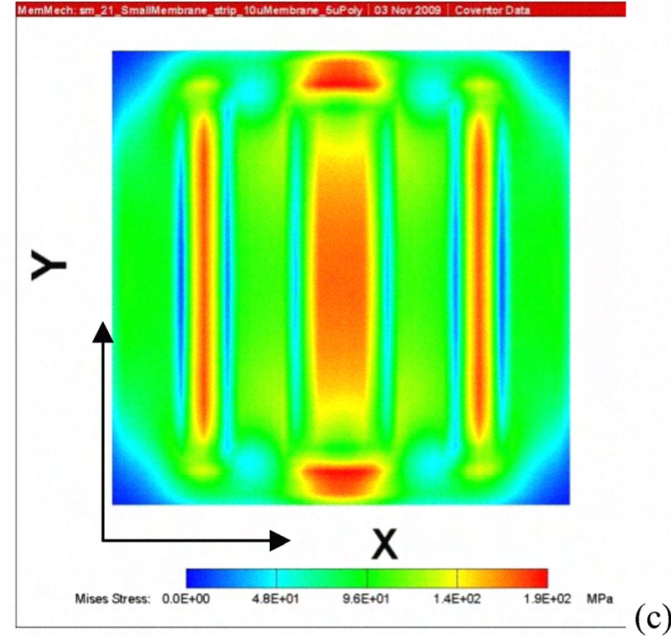

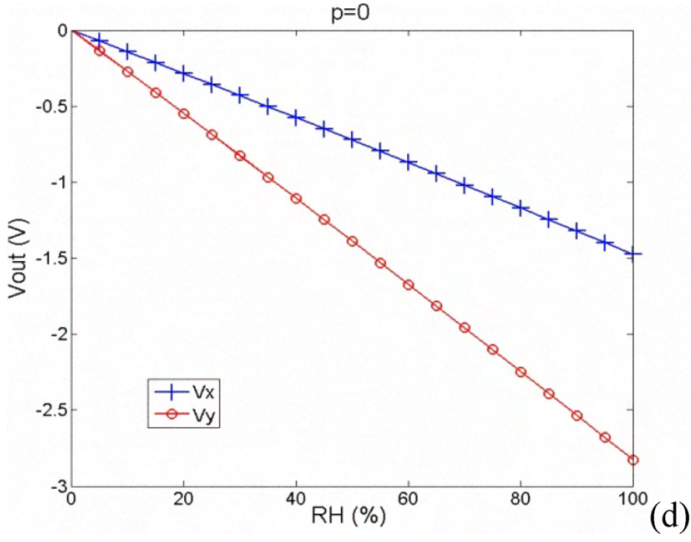

Figure 4, Mises stress on the membrane has different distributions caused by pressure and humidity. (a) Stress distribution when $\mathrm{p}=0.2 \mathrm{MPa}, \varphi=0 \%$. (b) Voltage output when $\varphi=0 \%$ and $p$ changes from 0 to $0.2 \mathrm{MPa}$. (c) Stress distribution when $p=0$ MPa, $\varphi=100 \%$. (d) Voltage output when $p=0$ MPa and $\varphi$ changes from 0 to $100 \%$.

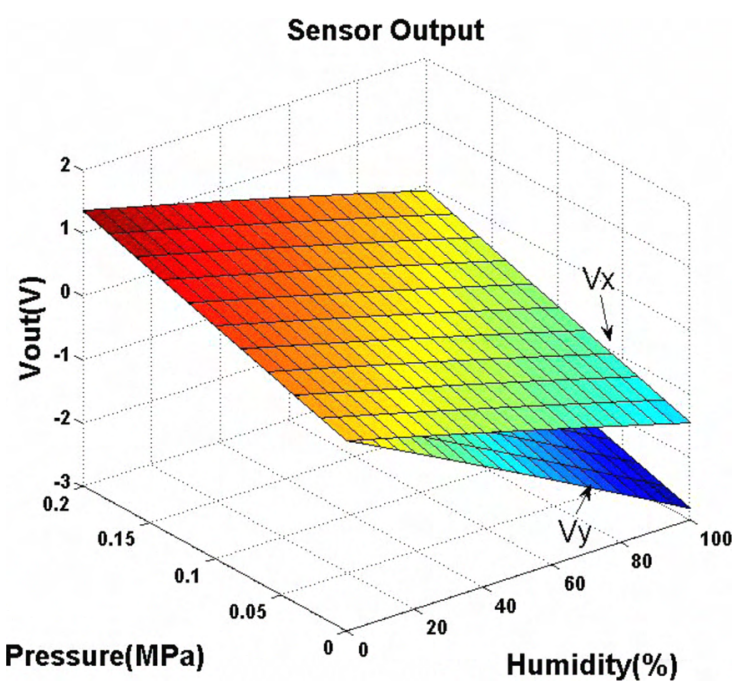

Figure 5, sensitivity map of the sensor output.

\section{BIST AND NETWORK SOLUTION}

A common issue in composite material structures is crosssensitivity to temperature, thus it is proposed to integrate a temperature measurement into the DMS. This could be achieved by the deposition of a thermal resistor on the substrate. A look-up table for temperature compensation can then be generated by sensor calibration. Similar work has been carried out on commercialized product [32].

Performing online built-in-self-test (BIST) of the DMS is much easier than with separate sensors, as only one main structure needs to be tested, although small modifications of the design might be required. The Bias-Superposition method is proposed to realise the real time test function [33]. There are two possible solutions: using thermal expansion or using an 
electrostatic force. The former requires additional heaters to be built in the membrane (Fig. 6(a)) to create thermal expansion of the membrane. The later requires an additional test electrode on the inside bottom of the sealed cavity (Fig. 6(b). A test voltage is then applied to this electrode, which will pull down the membrane. For both methods a small controlled deformation is superposed onto the membrane, together with the effect caused by RH and pressure. The test output is then separated from the measurement by a filter, and compared to the calibration coefficients (Fig. 6(c)). Applied test input could be close to or below noise level by using pseudo-random coding and crosscorrelation[34].
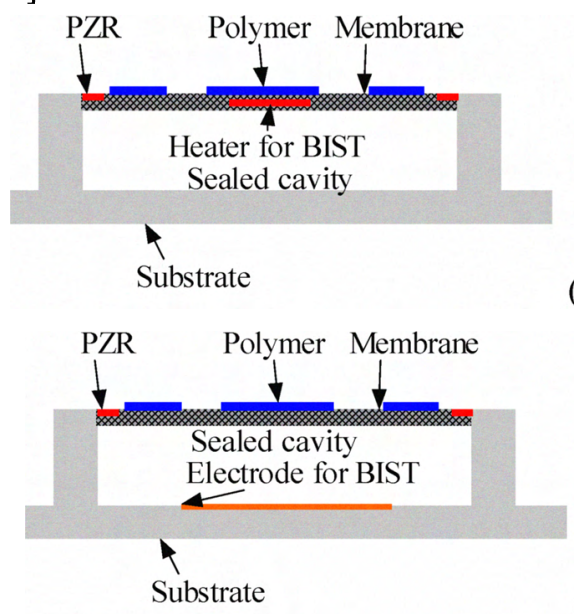

(a)

(b)

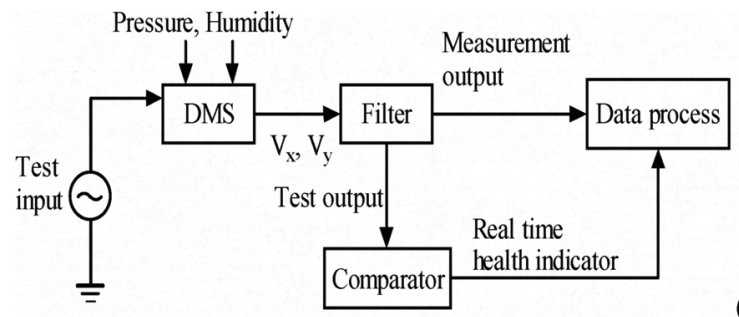

(c)

Figure 6, proposed online BIST solution by using (a) thermal expansion, and (b) electrostatic force. (c) Illustrates the test process. A test input is generated and applied to the sensor test component (heater or electrode). The deformation generated is monitored by the PZR, together with normal measurement of the pressure and the RH. A filter is then used to separate the test output from the measurement.

Based on the DMS we propose a Re-configurable Sensor Network (RCSN), that can be switched between Normal Operation and a Backup Modes. Fig. 7 shows an example of the structure of the network. A Local Data Processing Unit (LDPU) is used to collect and analyse the raw outputs from all the DMS in a RCSN node. The Following algorithm in the data processing unit will be implemented:

- Data pre-processing and sensor diagnosis: The measurement of $\mathrm{RH}$, pressure and temperature will be produced from the raw sensor output. Meanwhile the health state of each DMS will be evaluated by using the test output generated by the sensor's BIST function. The Confidence of Measurement (CoM) will then be passed to the internal data bus along with the measurements.

- If all the DMS's in the network are healthy, the data fusion algorithms average the output from each sensor to generate the final measurement of the environmental parameter being monitored. The RCSN is in its Normal Operation Mode.

- If any DMS fails the on-line test, the CoM from this sensor is low, thus will be discarded in the data fusion function. The RCSN is still in its Normal Operation Mode.

- If a significant percentage or even all the DMS's fail, the data fusion function forces the system into Backup Mode. Here the data fusion function utilizes the CoM coefficient to compute an output with the highest accuracy possible together with a global confidence metric. The measurement can only be used as a course indicator of condition, maintenance is then scheduled.

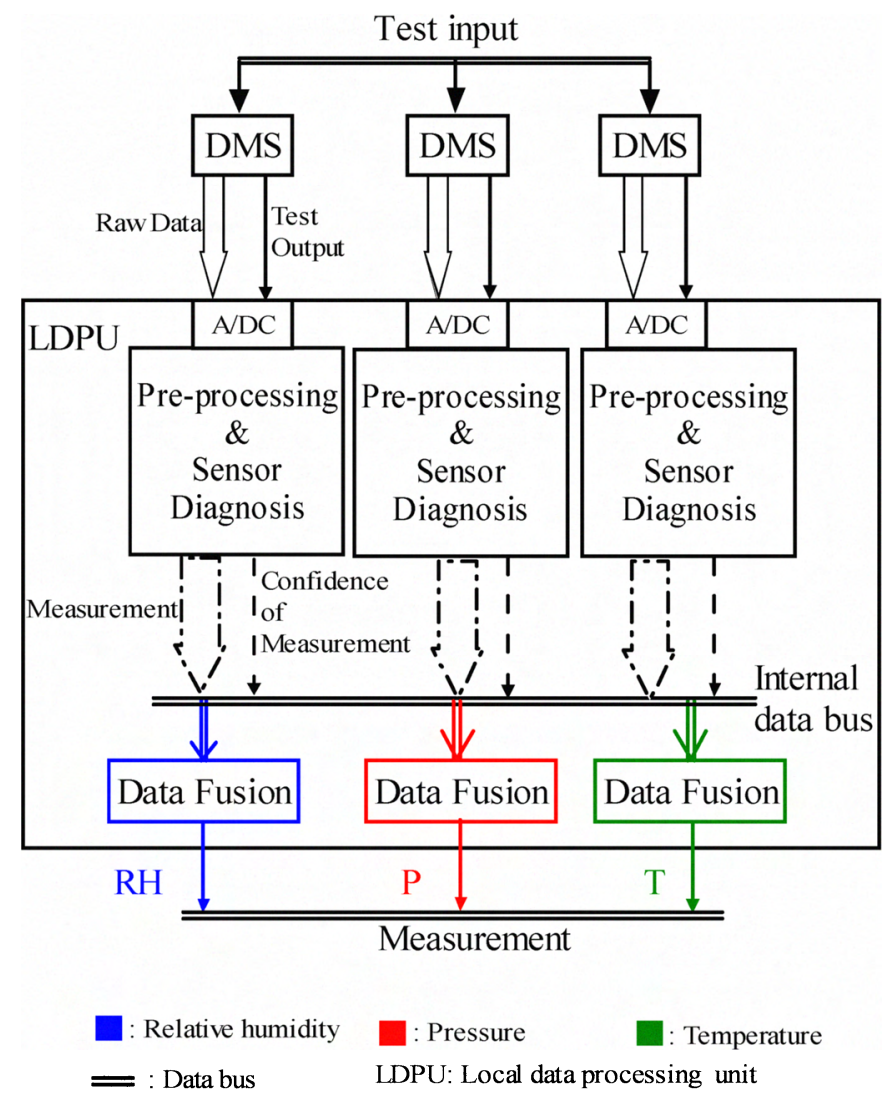

Figure 7, a re-configurable sensor network based on DMS

Compared with traditional redundancy, the RCSN can improve the reliability, and significantly reduce the number of sensors required within a HUMS (or any similar application). In the case presented here, if three physical parameters ( $\mathrm{RH}$, pressure and temperature) need to be monitored, redundancy will require at least 6 healthy sensors (without BIST feature) to generate the measurement, or 3 sensors with online BIST. 
RCSN requires only one healthy sensor. And in the extreme case where all sensors partially fail, RCSN would still be able to provide a courses reference measurement.

\section{$\checkmark$ CONCLUSION}

In this paper we have designed a dual mode humidity/pressure sensor with the potential to integrate a temperature function. Unlike other multi-function systems, the DMS is capable of measuring all physical parameters by a single physical device in the network. The final readout is generated by a post processing algorithm from the raw sensor data. The benefits include: reduced sensor size/cost due to packaging; simplified sensor structure, reduced external interconnect, improved reliability and yield and compatibility with BIST as only one test stimuli per device needs to be applied. We also proposed a re-configurable sensor network based on the multi function sensor, to exploit their advantages. The RCSN is particular useful for safety critical applications such as health and usage monitoring of large scale systems. The concept of multi-mode sensing and RCSN can be extended to many different types of MEMS devices due to their similarity in structure and fabrication process

\section{Acknowledgements}

The authors would like to thank the UK IeMRC, through the I-Health project and Ultra Electronics, through the Aerospace Wiring Test project for funding this work.

\section{References}

[1] J. McColl, "Overview of transmissions HUM performance in UK North Sea Helicopter Operation," in Institution of Mechanical Engineers Seminar S553, November 1997.

[2] P. M. Pawar and R. Ganguli, "Helicopter rotor health monitoring- a review," Proceedings of the Institution of Mechanical Engineers, Part G: Journal of Aerospace Engineering, vol. 221, pp. 631-647, 2007.

[3] G. P. Cleveland and C. Trammel, "Integrated Health and Usage Monitoring System for the SH-60B Helicopter," in 52nd Annual Forum of the American Helicopter Society, Washington, DC, USA, 1996, pp. 1767-1787.

[4] R. G. Carlson, S. D. Kershner, and R. A. Sewersky, "Sikorsky Health and Usage Monitoring System (HUMS) Program," in 52nd Annual Forum of the American Helicopter Society, Washington, DC, USA, 1996, pp. 1394-1406.

[5] J. R. C. Cole, "Health and Usage Monitoring of the EH101 Helicopter," in 52nd Annual Forum of the American Helicopter Society, Washington, DC, USA, 1996, pp. 417-420.

[6] R. S. Teal, M. L. Venuti, and A. Heather, "Health and usage monitoring on the international CH-47D," in 53rd Annual Forum of the American Helicopter Society, Virginia Beach, VA, USA, 1997, pp. 220-231.
[7] E. C. Robeson, "MH-47E Structural Usage Monitoring Systems (SUMS) Fleet," in 56th American Helicopter Society Forum, Virginia, USA, 2000, pp. 956-962.

[8] B. G. Cameron and P. Shathakumaran, "WAH-64 Apache HUMS Phase 1 Implementation," in 58th Annual Forum of the American Helicopter Society, Montreal, Canada, 2002, pp. 120-138.

[9] R. Dora, T. Baker, and R. Hess, "Applications of The IMD HUMS to the UH-60A Blackhawk," in 58th Annual Forum of the American Helicopter Society, Montreal, Canada, 2002, pp. 139-151.

[10] Y. Lu, R. A. Christ, T. A. Pucket, R. S. Teal, and B. Thompson, "AH-64D Apache Longbow Sructural Usage Monitoring System," in 58th Annual Forum of the American Helicopter Society, Montreal, Canada, 2002, pp. 152-162.

[11] J. E. Land, "HUMS-the benefits-past, present and future," in Aerospace Conference, 2001, IEEE Proceedings., 2001, pp. 3083-3094 vol.6.

[12] B. D. Larder, "Assessing the benefit of helicopter health and usage monitoring systems," in Aircraft Airborne Condition Monitoring, IEE Seminar on (Ref. No. 2003/10203), 2003, pp. 6/1-6/6.

[13] R. Heine and D. Barker, "Simplified terrain identification and component fatigue damage estimation model for use in a health and usage monitoring system," Microelectronics Reliability, vol. 47, pp. 1882-1888, 2007.

[14] C. J. Li and A. Ray, "Neural network representation of fatigue damage dynamics," in American Control Conference, 1995. Proceedings of the, 1995, pp. 3284-3288 vol.5.

[15] D. B. Jarrell and L. J. Bond, "Physics-based prognostics for optimizing plant operations," Sound \& Vibration, vol. 40, pp. 12-15, 2006.

[16] E. Schuster and K. C. Gross, "Multi-Frequency Sinusoidal Perturbation Method for Dynamic Characterization of Multi-Processor Computer Servers," Sun Microsytems May 2004.

[17] J. H. Yan and J. Lee, "Degradation Assessment and Fault Modes Classification Using Logistic Regression," Manufacturing Science and Engineering, vol. 127, pp. 912-914, November 2005.

[18] N. M. Vichare and M. G. Pecht, "Prognostics and health management of electronics," Components and Packaging Technologies, IEEE Transactions on, vol. 29, pp. 222-229, 2006.

[19] S. Neylon, A. Richardson, and H. Van Heeren, "Microsystems for structural health monitoring," NEXUS association http://eprints.lancs.ac.uk/26624/1/nexus SHM report Final.pdf, 2008.

[20] R. C. Muldoon, J. Gill, and L. D. Brock, "Integrated mechanical diagnostic (IMD) health and usage monitoring system (hums); an open system implementation case study," in Digital Avionics 
Systems Conference, 1999. Proceedings. 18th, 1999, pp. 9.B.4-1-9.B.4-8 vol.2.

[21] C. Furse and R. Haupt, "Down to The Wire," IEEE Spectrum, vol. 38, pp. 34-39, Feb 2001.

[22] R. Anchisini, G. Faglia, M. C. Gallazzi, G. Sberveglieri, and G. Zerbi, "Polyphosphazene membrane as a very sensitive resistive and capacitive humidity sensor," Sensors and Actuators B: Chemical, vol. 35, pp. 99-102, 1996.

[23] L. Gu, Q.-A. Huang, and M. Qin, "A novel capacitivetype humidity sensor using CMOS fabrication technology," Sensors and Actuators B: Chemical, vol. 99, pp. 491-498, 2004.

[24] P.-G. Su, C.-J. Ho, Y.-L. Sun, and I. C. Chen, "A micromachined resistive-type humidity sensor with a composite material as sensitive film," Sensors and Actuators B: Chemical, vol. 113, pp. 837-842, 2006.

[25] C.-L. Dai, "A capacitive humidity sensor integrated with micro heater and ring oscillator circuit fabricated by CMOS-MEMS technique," Sensors and Actuators B: Chemical, vol. 122, pp. 375-380, 2007.

[26] Y. Kim, B. Jung, H. Lee, H. Kim, K. Lee, and H. Park, "Capacitive humidity sensor design based on anodic aluminum oxide," Sensors and Actuators B: Chemical, vol. 141, pp. 441-446, 2009.

[27] P.-G. Su and C.-S. Wang, "Novel flexible resistivetype humidity sensor," Sensors and Actuators B: Chemical, vol. 123, pp. 1071-1076, 2007.

[28] K. Sager, A. Schroth, A. Nakladal, and G. Gerlach, "Humidity-dependent mechanical properties of polyimide films and their use for IC-compatible humidity sensors," Sensors and Actuators A: Physical, vol. 53, pp. 330-334, 1996.

[29] R. L. Gunter, W. D. Delinger, T. L. Porter, R. Stewart, and J. Reed, "Hydration level monitoring using embedded piezoresistive microcantilever sensors," Medical Engineering \& Physics, vol. 27, pp. 215-220, 2005.

[30] L.-T. Chen, C.-Y. Lee, and W.-H. Cheng, "MEMSbased humidity sensor with integrated temperature compensation mechanism," Sensors and Actuators A: Physical, vol. 147, pp. 522-528, 2008.

[31] R. Buchhold, A. Nakladal, G. Gerlach, and P. Neumann, "Design studies on piezoresistive humidity sensors," Sensors and Actuators B: Chemical, vol. 53, pp. 1-7, 1998.

[32] "DSHMX2000, HMX2000 Relative Humidity/Moisture Sensor," Hygrometrix Inc, Product Data Sheet 2000.

[33] C. Jeffrey, N. Dumas, Z. Xu, F. Mailly, F. Azais, P. Nouet, R. J. T. Bunyan, D. O. King, H. Mathias, J. P. Gilles, and A. M. D. Richardson, "Sensor testing through bias superposition," Sensors and Actuators A: Physical, vol. 136, pp. 441-455, 2007.

[34] N. Dumas, Z. Xu, K. Georgopoulos, R. J. T. Bunyan, and A. Richardson, "Online Testing of MEMS Based on Encoded Stimulus Superposition," Journal of
Electronic Testing, vol. 24, pp. 555-566, December, 2008. 